Revue interdisciplinaire des études canadiennes en

France

$80 \mid 2016$

Le Canada et la Grande Guerre

La morale à l'épreuve de la mondialisation : le rôle des valeurs morales et familiales dans la distinction idéologique du Canada en Amérique du Nord

Moral resistance versus globalization: the role of moral and family values in Canada's ideological distinction

Marie Moreau

OpenEdition

Journals

Édition électronique

URL : http://journals.openedition.org/eccs/712

DOI : $10.4000 /$ eccs. 712

ISSN : 2429-4667

Éditeur

Association française des études canadiennes (AFEC)

Édition imprimée

Date de publication : 1 juin 2016

Pagination : 171-186

ISSN : 0153-1700

Référence électronique

Marie Moreau, « La morale à l'épreuve de la mondialisation : le rôle des valeurs morales et familiales dans la distinction idéologique du Canada en Amérique du Nord », Études canadiennes / Canadian Studies [En ligne], 80 | 2016, mis en ligne le 01 juin 2017, consulté le 19 avril 2019. URL : http:// journals.openedition.org/eccs/712; DOI : 10.4000/eccs.712 


\title{
LA MORALE À L'ÉPREUVE DE LA MONDIALISATION : LE RÔLE DES VALEURS MORALES ET FAMILIALES DANS LA DISTINCTION IDÉOLOGIQUE DU CANADA EN AMÉRIQUE DU NORD
}

\author{
Marie MOREAU \\ Docteur de l'Université Sorbonne Nouvelle - Paris 3
}

\begin{abstract}
Alors que la mondialisation a contribué à une généralisation des modèles familiaux dans les sociétés occidentales, atténuant encore davantage les distinctions qui existent entre le Canada et les États-Unis, la réaction de l'opinion publique aux évolutions familiales indique qu'il existe malgré tout de profondes différences idéologiques entre ces deux sociétés. La relative faiblesse du jugement moral canadien semble être le signe d'une résistance structurelle et culturelle à l'influence de la moralité américaine, ce qui va à l'encontre d'une uniformisation idéologique nord-américaine.

Globalization has played a significant part in generalizing family structures in Western societies, reducing the number of distinctions between Canada and the United States. Yet, Canadians' and Americans' attitudes toward family changes point to deep ideological differences between the two societies. Moral judgment, although very important in the United States, does not seem particularly relevant in Canada, suggesting the existence of a moral resistance to American morality, a conclusion which goes against the theory of an ideological standardization of North America.
\end{abstract}

\section{Introduction}

La mondialisation, de par les échanges culturels et idéologiques qu'elle génère, a contribué à une généralisation des pratiques culturelles et des modèles sociétaux dans les sociétés occidentales. Les changements dans le marché du travail, dans les contextes économiques, dans les législations, dans l'éducation, qui résultent en partie des démarches globalisées d'un monde qui s'ouvre, ont eu un impact sur la famille, comme le note Bahira Sherif Trask:

Individuals and families are directly, and indirectly, affected by globalizing processes all over the world. [...] As we increasingly become integrated into new complex systems, individuals and their relationships, necessarily, are implicated in the process. [...] Globalization is accompanied by new transnational concepts about productivity, gender, work, nationalism, identity, familial relationships, and women's and children's rights (SHERIF TRASK 2008 : 4-5).

Les profondes évolutions des comportements familiaux et sexuels dont le Canada a été témoin depuis les années 1970, trouvent ainsi un écho dans la 


\section{Marie MOREAU}

plupart des pays occidentaux. Cependant, il est intéressant de constater que si les modèles familiaux s'exportent au gré de la mondialisation, produisant une certaine harmonisation des modes de vie familiaux dans le monde occidental, les valeurs morales, quant à elles, semblent moins perméables. Les différentes attitudes de l'opinion publique à l'égard de ces évolutions montrent en effet que la similarité des comportements peut cacher des différences idéologiques, esquissant une position particulière pour le Canada, positionné entre la grande permissivité morale européenne et le conservatisme moral américain.

Cette position d'entre-deux pose la question de l'impact de la très puissante moralité américaine sur les valeurs canadiennes dans un contexte de mondialisation qui accentue encore l'influence culturelle et idéologique qu'exerce le géant sur son voisin du Nord. Alors que leur proximité linguistique, géographique et culturelle tend déjà naturellement à atténuer les distinctions qui existent entre eux (BALTHAZAR 2007 : 134), la multiplication des échanges culturels, médiatiques et économiques entre le Canada et les États-Unis renforce encore l'impression d'une uniformisation nord-américaine. Pourtant, le jugement de l'opinion publique canadienne et américaine sur les évolutions familiales diffère nettement, et ce, malgré l'influence morale à laquelle la mondialisation a exposé les Canadiens, notamment à travers la culture évangélique américaine.

Dans un contexte de mondialisation où la question de l'influence culturelle et idéologique des pays les uns envers les autres est d'autant plus pertinente dans le cas du duo Canada/États-Unis, la distinction morale exprimée par l'opinion publique canadienne à l'égard des questions familiales semble aller à l'encontre de l'apparente uniformisation des modèles sociétaux, et familiaux en particulier. Dans cette entreprise comparatiste, il parait, dans un premier temps, nécessaire d'analyser les données de la statistique officielle, des Nations Unies et de l'Organisation de Coopération et de Développement Economique (OCDE), qui permettent une étude longitudinale tout en garantissant la comparabilité des données entre les pays, afin de replacer les évolutions familiales canadiennes parmi les tendances américaines et européennes. Ensuite, les enquêtes d'opinion sur la moralité nous indiqueront la position du Canada en termes de permissivité morale sur ces questions, ce qui nous poussera enfin à interroger les limites de l'influence de la morale américaine sur les questions familiales canadiennes. 


\section{LA MORALE À L’ÉPREUVE DE LA MONDIALISATION}

\section{Les évolutions familiales canadiennes, reflet d'un processus globalisé}

$\mathrm{Au}$ cours des quarante dernières années, d'importants changements économiques, sociaux, contextuels et législatifs ont permis de libérer les comportements familiaux du carcan de la famille traditionnelle. Parmi les évolutions familiales, la diversification des formes conjugales et la modification des comportements liés à la fécondité ont particulièrement refaçonné le paysage sociodémographique des sociétés occidentales. Si les causes de ces évolutions sont multiples et bien souvent imbriquées les unes dans les autres, certaines avancées législatives ont un impact direct sur les comportements, à l'image de la Loi sur le divorce de 1968, puis de l'entrée en vigueur des amendements de 1986, qui se traduisent par une recrudescence immédiate du nombre de divorces au Canada. De 1,38 divorces pour 1000 Canadiens en 1971, le taux brut de divortialité a augmenté pour se stabiliser à 2,1 pour 1000 en 2008 (date des dernières données disponibles pour le Canada $)^{1}$. Le Canada s'inscrit alors dans une tendance commune aux autres pays occidentaux ; on note toutefois une nette différence par rapport aux ÉtatsUnis, où l'on relève 3,5 divorces pour 1000 Américains la même année ${ }^{2}$. En comparaison, le comportement du Canada ressemble davantage à celui de certains pays européens comme la France (2,1 divorces pour 1000 habitants), les Pays-Bas $(2,0)$, la Norvège $(2,1)$, l'Allemagne, $(2,3)$, l'Autriche $(2,4)$, le Royaume-Uni $(2,0)$ ou encore la Finlande $(2,5)$.

En parallèle, dans la très grande majorité des sociétés occidentales, le taux de nuptialité a considérablement baissé à mesure que son statut de seul cadre socialement et légalement acceptable pour la vie conjugale et parentale a été remis en cause. Au Canada, on dénombrait 8,9 mariages pour 1000 habitants en 1971 alors qu'on en compte 4,4 pour 1000 en $2008^{3}$. La tendance au mariage est également à la baisse aux États-Unis, toutefois les Américains continuent à se marier dans des proportions beaucoup plus importantes que leurs voisins (et que les autres pays développés) : leur taux brut de nuptialité se

\footnotetext{
${ }^{1}$ NATIONS UNIES, 1992, Département des affaires économiques et sociales internationales, Annuaire démographique 1990, New York, tableau 34, http://unstats.un.org/unsd/demographic/products/dyb/dybsets/1990\%20DYB.pdf (consulté le 22 octobre 2015). NATIONS UNIES, 2011, Département des affaires économiques et sociales internationales, Annuaire démographique 2011, New York, tableau 25,

http://unstats.un.org/unsd/demographic/products/dyb/dyb2011.htm (consulté le 22 octobre 2015)

${ }^{2}$ Les données américaines sur le divorce pour cette année ne figurent pas dans l'annuaire démographique des Nations Unies mais sont publiées par le Center for Disease Control and Prevention Center (CDC) et par l'OCDE.

${ }^{3}$ NATIONS UNIES, 1992 : tableau 24 ; NATIONS UNIES, 2011 : tableau 23.
} 


\section{Marie MOREAU}

situe à 7,1 pour 1000 habitants en 2008 (par rapport à 10,5 en 1971). En comparaison, la nuptialité canadienne est plutôt à rapprocher des tendances européennes comme celles de la France (4,2 mariages pour 1000 habitants), de la Belgique $(4,3)$, de l'Allemagne $(4,6)$, des Pays-Bas $(4,5)$, ou du RoyaumeUni $(4,4)$. Les données américaines, pour leur part, se comparent plutôt à celles de pays comme la Géorgie $(7,2)$ ou l'Albanie $(6,7)$.

Le recul du mariage semble se faire au profit de l'union libre, qui connaît un grand succès dans les sociétés occidentales et s'est rapidement imposée comme forme familiale à part entière. Au Canada, c'est dans le cadre du recensement de 1981 que l'on dénombre pour la première fois les couples en union libre ; les conjoints de fait représentaient alors 5,6\% de toutes les familles de recensement. Depuis, la proportion de couples en union libre a connu une augmentation rapide et concerne $16,7 \%$ des ménages en $2011 .^{4}$ Les données recueillies par l'OCDE indiquent que l'union libre s'est également fait une place parmi les familles américaines, cependant elle reste moins importante qu'au Canada. En 2011, dans la tranche d'âge des 20 à 34 ans, particulièrement susceptibles de débuter leur vie conjugale et parentale, $21,8 \%$ des Canadiens déclarent vivre en union libre alors que ce n'est le cas que de $12,2 \%$ des Américains $^{5}$. Il convient tout de même de noter que les personnes comptabilisées comme vivant en union libre sont celles qui ne sont ni mariées ni engagées dans un autre type d'union civile, or la valeur accordée à l'union libre et aux unions civiles diffère selon les pays ${ }^{6}$, les comparaisons sont donc à prendre avec précaution. Notons tout de même que la nette différence entre le recours à l'union libre au Canada et aux États-Unis s'explique en partie par le nombre très élevé de conjoints de fait dans la province du Québec, où 31,5\% des couples vivent ensemble sans être mariés en 2011, ce qui représente plus du double de la moyenne de $12,1 \%$ observée dans les autres provinces. ${ }^{7}$ Le

\footnotetext{
${ }^{4}$ STATISTIQUE Canada (2012a), Ministère de l'Industrie, Gouvernement du Canada, Cinquante ans de familles au Canada : 1961 à 2011, 98-312-X2011003, Ottawa, p. 2.

${ }^{5}$ OCDE (Organisation de coopération et de développement économique) (2015a), Base de données sur la famille 2014, http://www.oecd.org/fr/els/famille/basededonnees.htm\#composition. Pour les données sur le divorce en 1970 et 2008, on regardera le feuillet SF3.1 de 2014 : http://www.oecd.org/els/family/SF3 1_Marriage_and_divorce_rate_Jan2014.pdf

${ }^{6}$ Les couples canadiens en union libre ont par exemple à peu près les mêmes avantages que les couples mariés en termes de fiscalité, de reconnaissance publique et administrative, ou même d'immigration. Le choix de l'union civile au Canada ne se fait donc par exemple pas dans les mêmes termes que celui du Pacte civil de solidarité (PACS) français, qui répond à un besoin de reconnaissance fiscale et administrative des couples cohabitants français.

${ }^{7}$ STATISTIQUE CANADA (2012b), Ministère de l'Industrie, Gouvernement du Canada, Portrait des familles et situation des particuliers dans les ménages au Canada. Recensement de la population de 2011, 98-312-X2011001, Ottawa, p.7.
} 


\section{LA MORALE À L’ÉPREUVE DE LA MONDIALISATION}

Québec dépasse ainsi la Suède ( $29 \%$ en 2010$)$, la Finlande (24,7\% en 2010$)$ et la Norvège $(23,9 \%$ en 2011), réputés être les pays les plus ouverts à cette structure familiale.

Outre la modification des formes de vie conjugale, l'évolution dans les comportements de fécondité est l'un des changements qui a marqué l'évolution des sociétés et des familles occidentales au cours de ces quarante dernières années. Le taux de fertilité pour la moyenne des pays de l'OCDE est ainsi passé de 2,76 enfants par femme en 1970 à 1,67 en $2013 .{ }^{8}$ La baisse généralisée des naissances n'a pas épargné le Canada, qui enregistre une chute de 2,33 enfants par femme en 1970 à 1,61 en 2013, pas plus que les États-Unis, qui passent de 2,48 enfants par femme en 1970 à 1,86 en 2013. Les chiffres du Canada le rapprochent encore une fois de certains pays européens (1,67 au Danemark ; 1,68 aux Pays-Bas) tout en le situant tout de même au-dessus de la moyenne européenne établie à 1,52 enfants par femme. Les États-Unis, pour leur part, se situent tout à fait dans le haut de cette moyenne et sont uniquement dépassés par l'Irlande $(1,96$ enfants par femme) et par la France $(1,98)$.

Les pratiques culturelles, les valeurs sociales, les modèles économiques, les représentations médiatiques, les influences législatives dont la mondialisation se fait le vecteur entre les différentes sociétés occidentales ont contribué à une généralisation des modèles sociétaux, à laquelle les comportements familiaux n'échappent pas. Le Canada et les États-Unis se retrouvent ainsi autour d'évolutions familiales communes, qui pourtant, au lieu de les rapprocher, permettent, à la lumière du traitement que ces sociétés leur réservent, de mettre en avant leurs différences.

\section{La dimension morale dans les questions familiales canadiennes et américaines}

Si la grande majorité des sociétés occidentales sont affectées par la diversification des comportements familiaux, toutes n'adoptent la même attitude à leur égard. La remise en cause du modèle traditionnel, autour duquel se cristallisaient d'autres valeurs plus larges comme diverses formes d'autorité ou encore la répartition différentiée des rôles selon les sexes, nourrit une certaine appréhension chez un public plus conservateur. Parce que la famille occupe une place centrale, elle tend à être considérée par les sociétés plus

\footnotetext{
${ }^{8}$ OCDE (2015b), «Fertility rates, 1970-2014», disponible sur le site internet de l'OCDE à l'adresse suivante : https://data.oecd.org/pop/fertility-rates.htm (consulté le 18 octobre 2015)
} 


\section{Marie MOREAU}

traditionnelles comme garante de la vertu; Kathleen Sands écrit d'ailleurs à propos des États-Unis : «Everywhere, "family" is the icon of goodness and the standard of value » (SANDS 2000: 7). Dans ce contexte, les attitudes de l'opinion publique canadienne et américaine sur le caractère moral des évolutions familiales devraient être éclairantes. Deux sondages menés la même année par les firmes Angus Reid et Pew Research Center sur la moralité permettent de mettre en avant des différences entre les États-Unis et le Canada que la relative similarité des indicateurs sociodémographiques ne permet pas de déceler. En 2013, Angus Reid invite des Canadiens, des Américains et des Britanniques à se prononcer sur la moralité de certains sujets ayant un fort pouvoir divisif auprès de l'opinion publique, tels que l'avortement, la polygamie, la pornographie, ou encore la recherche sur les cellulles souches. La consigne est la suivante : « Regardless of whether or not you think each of the following issues should be legal, please indicate whether you personally believe they are morally acceptable or morally wrong ».

L'exemple du divorce est à ce titre particulièrement intéressant; dans la mesure où les Américains ont un recours au divorce beaucoup plus important que les Canadiens, il serait a priori logique de penser que cette pratique est mieux acceptée aux États-Unis qu'au Canada. Or, seulement $65 \%$ des personnes interrogées aux États-Unis jugent que le divorce est moralement acceptable, alors que c'est le sentiment de $80 \%$ des Canadiens sondés (comme $79 \%$ des Britanniques). L'enquête "Global views on Morality" menée par le Pew Research Center en 2013 auprès de 40 pays apporte un autre éclairage sur cette question'. Les participants doivent cette fois dire si les sujets qu'on leur présente leur semblent moralement acceptables, moralement inacceptables, ou s'il ne s'agit pas d'une question morale. Seulement 9\% des Canadiens considèrent qu'il est moralement inacceptable de divorcer. A titre de comparaison, ceci place le Canada un peu derrière la France et l'Allemagne (respectivement $5 \%$ et $7 \%$ ), et au même niveau que la Grande-Bretagne. Aux États-Unis, en revanche, $22 \%$ des personnes interrogées pensent que le divorce est moralement répréhensible, une condamnation en proportion similaire à celle de la Russie et de la Pologne.

L'apparente contradiction entre le recours plus fréquent des Américains au divorce et la condamnation morale plus sévère qu'ils lui adressent pourtant semble pointer vers un fort attachement à l'institution du mariage. A l'inverse, la plus grande aisance des Canadiens avec les nouvelles

${ }^{9}$ PEW RESEARCH CENTER (2014), Global Views on Morality, 2013,

http://www.pewglobal.org/2014/04/15/global-morality/ (consulté le 18 février 2015). 


\section{LA MORALE À L’ÉPREUVE DE LA MONDIALISATION}

structures familiales, comme en témoigne leur plus grande propension à choisir le concubinage, se traduit également par une plus grande acceptation morale de ce mode de vie conjugale comme structure familiale à part entière. En effet, 78\% des Canadiens sondés par Angus Reid pensent qu'il est moralement acceptable d'avoir un enfant hors mariage (comme $74 \%$ des Britanniques) alors que seulement la moitié des Américains (53\%) trouvent qu'il est moral de fonder une famille en dehors du mariage. A la condamnation moins sévère des structures familiales qui mettent à mal l'institution du mariage, semble s'ajouter un jugement plus permissif de l'ensemble des comportements sexuels de la part des Canadiens. Ainsi, $83 \%$ des Canadiens interrogés par Angus Reid pensent qu'il est moralement acceptable pour un homme et une femme qui ne sont pas mariés d'avoir des relations sexuelles (à l'image de $82 \%$ des Britanniques), alors que c'est le cas de seulement $59 \%$ des Américains. Confrontés à la même question posée par le Pew Research Center, la proportion des Américains qui réprouvent les relations sexuelles hors mariage $(30 \%)$ est deux fois plus importante que celle des Canadiens (15\%). A travers cette question, la position particulière d'un Canada situé entre l'Europe et l'Amérique ressort clairement puisqu'il se place entre la permissivité morale des pays européens (en France, en Allemagne ou en Espagne par exemple, la condamnation de la sexualité hors mariage oscille entre $6 \%$ et $8 \%$ ), et le conservatisme moral américain (qui, sur ce sujet, a une proportion de condamnation équivalente à celle de la Russie).

C'est la question de l'homosexualité, parce qu'elle mêle famille, sexualité et morale, qui offre les résultats les plus divisés. La proportion de répondants qui trouvent l'homosexualité inacceptable moralement est deux fois et demi plus faible au Canada (15\%) qu'aux États-Unis (37\%) selon le Pew Research Center. Ceci place une fois de plus les Canadiens dans cette position d'entre-deux : entre la tolérance morale de certains pays européens $(6 \%$ des Espagnols, $8 \%$ des Allemands, $14 \%$ des Français interrogés réprouvent l'homosexualité) et le traditionnalisme des États-Unis (qui se rapprochent davantage de certains pays d'Amérique latine, comme le Chili, le Brésil et le Mexique, où la condamnation de l'homosexualité s'élève respectivement à $32 \%, 39 \%$ et $40 \%)$.

Le regard nettement moins conservateur des Canadiens sur les nouveaux comportements familiaux et sexuels a sans doute beaucoup à voir avec la place moins importante qu'ils octroient à la morale dans leur jugement. Ainsi, près de la moitié des Canadiens déclarent au Pew Research Center que la question du divorce (47\%) et celle de l'homosexualité (50\%) ne sont pas des questions morales, comparativement à seulement $35 \%$ des Américains sur ces 


\section{Marie MOREAU}

deux sujets. Il en va de même pour la sexualité hors mariage, que $47 \%$ des Canadiens face à seulement $36 \%$ des Américains n'envisagent pas sous l'angle moral. Enfin, sur une question aussi sensible que l'avortement qui cristallise bien souvent des arguments d'ordre moral, 37\% de la population canadienne interrogée considère que ce sujet ne relève pas de la morale, quand ce n'est le cas que de $23 \%$ des sondés américains.

Non seulement l'opinion publique canadienne est beaucoup moins susceptible que l'opinion américaine d'utiliser la morale comme critère de jugement pour les affaires familiales, sexuelles et sociales, mais en outre le Canada se montre beaucoup plus permissif que son voisin d'un point de vue moral, se rapprochant souvent davantage des tendances européennes qu'américaines. Dès lors, la place accordée à la morale et le rôle qu'elle joue au sein de chacune de ces deux sociétés semblent opérer une distinction idéologique fondamentale dans leur fonctionnement social et familial.

\section{L'exportation de la morale américaine au Canada et ses limites}

Si l'opinion canadienne, malgré l'influence religieuse, sociale et culturelle américaine à laquelle elle est soumise, n'adopte pas le jugement moral de l'opinion américaine, c'est que la morale n'a pas la même valeur dans la société canadienne. Pour Seymour Martin Lipset, les causes sont en partie à chercher dans les principes fondateurs même de ces deux sociétés, qui auraient fait de la morale une institution aux États-Unis, lui conférant alors un rôle de régulateur de la vie sociale qu'elle n'occupe pas au Canada (LIPSET 1996). C'est une idée que partage Gertrude Himmerlfarb, qui reprend les propos de John Adams («Our constitution was made only for a moral and religious people. It is wholly inadequate to the government of any other ») et de George Washington (« Of all dispositions and habits which lead to political prosperity, religion and morality are indispensable supports ») afin de montrer que l'ancrage de la morale dans le système de gouvernance pour garantir le bon ordre social était une intention des architectes de la Constitution américaine (HIMMELFARB 2001:86). Lipset ajoute que c'est paradoxalement la séparation de l'Église et de l'État qui a indirectement donné un rôle central à la morale en l'institutionnalisant (LIPSET 1996). La responsabilité qui a incombé à la religion américaine de maintenir l'ordre moral et social lui a imposé d'adopter une ligne de conduite plus stricte, tandis que la prise en charge de la religion par l'État canadien a permis à la société canadienne de faire évoluer sa religion au gré de la modernisation des institutions, et de graduellement lui accorder moins d'importance. Puisque la place de la morale semble intimement 
liée à celle de la religion, on ne s'étonnera pas que Lipset y cherche d'autres facteurs pouvant expliquer la différence dans les attitudes canadiennes et américaines. Ainsi, la philosophie religieuse même du protestantisme américain, parce qu'elle est sous-tendue par l'idée de perfectibilité de l'humanité et par l'obligation d'éviter le péché, pousse selon lui ses fidèles à adopter la morale comme ligne directrice dans tous leurs comportements et jugements, alors que les Églises qui prédominent en Europe ou au Canada ont accepté la faiblesse inhérente des hommes et leur impossibilité à échapper à l'erreur (LIPSET 1991: 76). Il met également en cause les origines des religions aux États-Unis et au Canada :

The religious traditions of Protestant "dissent" have called on Americans to be moralistic, to follow their conscience with an unequivocal emphasis not to be found in countries whose predominant denominations have evolved from state churches (LIPSET 1996 : 63).

Dès lors, Lipset en conclut que la combinaison de ces éléments donne une importance fondamentale à la morale, qui devient alors une référence pour juger les questions sociales aux États-Unis, ce que l'on ne retrouve pas dans la même mesure au Canada.

Pourtant, entre ces deux sociétés nord-américaines où le jeu des influences dépasse de beaucoup l'interdépendance économique, la morale américaine, à l'image d'autres objets culturels ou idéologiques, s'exporte au Canada. La mondialisation a notamment exposé les Canadiens à la culture évangélique américaine à travers ses nombreuses productions médiatiques et culturelles (livres, magazines, émissions de télévision et de radio) et l'influence idéologique américaine commence à porter ses fruits. Les Canadiens ont ainsi commencé à rejoindre des dénominations évangéliques basées aux États-Unis ou à développer des branches canadiennes, comme l'illustre l'ouverture, en 1983, d'une branche canadienne de l'organisation évangélique Focus on the Family. Cette organisation très conservatrice milite pour la conservation des valeurs et des modèles familiaux traditionnels et moraux, et se mobilise à chaque fois qu'une de ces questions se retrouve au centre des débats. On peut également sentir les prémices d'une implication des évangéliques en politique lorsqu'en 2000, Stockwell Day, qui ne cache pas son affiliation évangélique, emprunte les tactiques électorales de la Droite religieuse américaine et prend la tête de l'Alliance canadienne, alors parti de l'opposition. L'ancien président de la branche canadienne de Focus on the Family, Darrel Reid, a d'ailleurs occupé 


\section{Marie MOREAU}

d'importantes fonctions au sein du cabinet du Premier ministre Stephen Harper entre 2007 et 2010 .

Dans la mesure où l'évangélisme élève les croyances religieuses traditionnelles au rang de vérités morales et sociales absolues, il parait a priori logique de penser qu'une société influencée par des courants évangéliques fasse preuve de jugements moraux plus intransigeants sur des questions sociales dont on peut considérer qu'elles s'éloignent des schémas traditionnels. Il est difficile d'obtenir des chiffres précis sur le nombre de Canadiens et d'Américains qui adhèrent à des mouvements évangéliques, mais il est certain que les évangéliques sont moins importants au Canada qu'aux États-Unis. Les estimations varient généralement entre 10 et $12 \%$ de la population canadienne, et 25 et $35 \%$ de la population américaine (BEAN et al. 2008). En 2008, les chercheurs Rosson et Fields estiment plus précisément que les évangéliques représentent $10,77 \%$ de la population canadienne et $32,49 \%$ de la population américaine (ROSSON et FIELDS 2008) ; l'influence de l'évangélisme est donc encore limitée au nord de la frontière.

Les liens entre l'importance de la morale dans les jugements sur les questions familiales et la présence d'évangéliques au sein de ces deux sociétés a été l'objet d'une enquête publiée par Hoover, Martinez, Reimer et Wald en 2002. En cherchant à savoir si les évangéliques accordent plus d'importance à la morale que le reste de la population, cette enquête a mis en lumière plusieurs différences intéressantes entre le Canada et les États-Unis. Tout d'abord, ils confirment que les évangéliques sont plus moralistes dans les attitudes familiales que le reste de la population. Dans la mesure où l'évangélisme est moins présent au Canada qu'aux États-Unis, il est donc logique que le jugement moral soit moins important au nord qu'au sud de la frontière. Il ressort d'ailleurs également de l'étude que l'ensemble de la population canadienne est moins moraliste que l'ensemble de la population américaine. Enfin, curieusement, les chercheurs établissent clairement que les évangéliques canadiens sont bien moins moralistes que les évangéliques américains. Il y a donc véritablement une résistance au moralisme au Canada, même parmi les populations qui y sont plus enclines. Les auteurs de l'étude expliquent en partie la relative faiblesse du rigorisme moral dans l'évangélisme canadien par la présence mineure de fondamentalistes au sein du mouvement canadien en regard du courant américain. Leurs directives strictement morales sur les questions familiales influencent donc moins les évangéliques canadiens, ainsi que l'ensemble de la population canadienne (HOOVER et al. 2002). 


\section{LA MORALE À L’ÉPREUVE DE LA MONDIALISATION}

Par ailleurs, l'évangélisme canadien, parce qu'il est inspiré de l'évangélisme britannique, bien plus accommodant, et qu'il ne s'est pas totalement éloigné du protestantisme, conserve des attitudes moins empreintes d'absolu moral que celles de l'évangélisme américain qui, en se coupant des églises protestantes établies, s'est retranché dans des attitudes plus radicales, faisant du moralisme une de ses marques distinctives (BEAN et al. 2008). En outre, les origines de ces deux sociétés expliquent en partie la résistance à l'influence du moralisme et de l'évangélisme. Aux États-Unis, les évangéliques se font entendre sur tout le territoire car ils peuvent mobiliser une grande partie des Américains autour d'origines et de valeurs protestantes communes dans lesquelles ceux-ci se retrouvent. A l'inverse, la division religieuse et culturelle au Canada a empêché le moralisme évangélique de trouver un écho soutenu auprès de l'ensemble de la population (BEAN et al. 2008). Le Québec et les catholiques se sont maintenus en dehors des courants évangéliques, protestants, et il est intéressant à présent de constater que les Québécois ont un degré de moralisme plus faible à l'égard des questions familiales que leurs compatriotes et que leurs voisins (HOOVER et al. 2002).

En effet, si jusqu'à la fin des années 1960 le Québec s'illustrait surtout par des comportements sociodémographiques et des valeurs morales fortement empreints du conservatisme social et moral imposé par l'Église catholique, la province à majorité francophone se démarque à présent du reste du Canada par son ouverture à l'égard des questions familiales. Cette permissivité se traduit par une adoption plus importante que dans le reste du Canada des structures familiales qui dévient de la norme traditionnelle qu'est l'institution du mariage, et par une condamnation morale beaucoup plus faible sur ces questions que le reste du Canada, et a fortiori que les États-Unis. Le dernier recensement canadien rapporte que c'est au Québec que le recours au mariage est le plus faible, avec 51,9\% des ménages composés de couples mariés en 2011 comparativement à $71 \%$ en moyenne pour les autres provinces ${ }^{10}$. Le Québec se trouve donc naturellement être la province qui a le plus tendance à envisager l'union libre comme une véritable structure familiale (parentale, et non seulement conjugale) alternative au mariage : 37,8\% des enfants québécois âgés de 14 ans et moins vivent avec des parents en union libre en 2011 par rapport à $16,3 \%$ des enfants dans l'ensemble du Canada ${ }^{11}$.

\footnotetext{
${ }^{10}$ STATISTIQUE CANADA, 2012b : 6

${ }^{11}$ STATISTIQUE CANADA, 2012b : 15
} 


\section{Marie MOREAU}

L'attachement moins fort aux normes traditionnelles que laissent transparaitre les choix familiaux des Québécois, nous invite à penser que la morale occupe moins de place dans leurs jugements. C'est ce que confirmait déjà une enquête de 1981 dans laquelle les Québécois se démarquaient des Canadiens et des Américains dans le refus du lien entre la morale et la sexualité: 34\% des Québécois pensaient que les rapports sexuels devaient être encadrés par des règles morales, quand c'était l'avis de $49 \%$ des Canadiens anglophones et de 51\% des Américains (LIPSET 1986). Les sondages sur la moralité menés en 2013 par Angus Reid et le Pew Research Center ne permettent pas de discerner le Québec des autres provinces, toutefois la même enquête menée par Angus Reid au Canada en 2007 opérait cette distinction. Les résultats faisaient état d'une faible condamnation morale québécoise à l'égard des nouvelles configurations familiales, à l'image de l'union libre comme forme de vie conjugale ( $8 \%$ des Québécois trouvent moralement répréhensibles les relations sexuelles entre deux adultes non mariés, comparativement à $15 \%$ en moyenne pour l'ensemble des Canadiens), ou comme structure parentale (8\% des Québécois condamnent moralement le fait d'avoir un enfant en dehors des liens du mariage quand c'est le cas de $17 \%$ en moyenne à l'échelle nationale). Moins attachés au mariage, les Québécois se montrent également plus compréhensifs sur la question du divorce (8\% des Québécois le réprouvent par rapport à $12 \%$ pour l'ensemble du Canada). L'influence du Québec n'est donc pas à négliger dans la différenciation idéologique du Canada par rapport aux États-Unis, car le Québec, de par ses différences linguistiques, religieuses, historiques et culturelles avec les États-Unis, offre plus de résistance à la morale américaine et est plus imperméable aux effets de la mondialisation culturelle en provenance des États-Unis.

\section{Conclusion}

La proximité géographique, linguistique, culturelle, économique, et médiatique du Canada et des États-Unis fait dire à Louis Balthazar qu'ils sont « les deux pays les plus intégrés l'un à l'autre dans le monde » (BALTHAZAR 2007 : 134). Cette impression de parallélisme est encore renforcée par une apparente similitude des comportements individuels et familiaux des Canadiens et des Américains, dont l'évolution s'inscrit dans une tendance généralisée aux pays occidentaux. Les Canadiens, à l'image des autres occidentaux, peuvent se reconnaître dans les productions médiatiques américaines mettant en scène des femmes qui travaillent et des familles divorcées, recomposées, homoparentales, monoparentales ou vivant en union libre et comptant un petit nombre d'enfants. 


\section{LA MORALE À L’ÉPREUVE DE LA MONDIALISATION}

Pourtant, si cette apparente homogénéité renforce l'idée d'une harmonisation nord-américaine, certaines différences dans les indicateurs sociodémographiques liés à la famille sont autant d'indices que les valeurs familiales canadiennes et américaines divergent sur certains points. L'introduction de la dimension morale dans les questions familiales confirme que les Américains et les Canadiens n'acceptent pas de la même manière les évolutions familiales qui ont touché leur société, et est révélatrice de la place différente qui lui est accordée au sein de ces sociétés. Parce que la société américaine accorde une telle importance à la moralité, Lipset écrit que les Américains sont des «utopian moralists who press hard to institutionalize virtue, to destroy evil people, and eliminate wicked institutions and practices » (LIPSET 1996 : 63). Les Canadiens, à l'inverse, sont plus permissifs du point de vue moral, et ont davantage tendance à considérer les questions familiales comme des questions sociales et non morales.

Dans cette distinction, le rôle joué par le Québec ne peut être négligé. A cet égard, les sociologues Céline Le Bourdais et Nicole Marcil-Gratton font le constat suivant: "without Québec's specific conservatism in the past and today's tremendous reversal of its behaviors, Canada's kinship with the US would be far more obvious » (LE BOURDAIS et MARCIL-GRATTON 1996 : 416). S'il est difficile d'identifier une raison unique à cette particularité des attitudes et des comportements familiaux et moraux des Québécois, le rôle de la Révolution tranquille, qui les aurait poussé à abandonner la morale comme ligne directrice, semble incontestable. Laplante, Miller et Malherbe, qui s'étonnent que la seule province canadienne à majorité catholique se trouve pourtant être celle dans laquelle on organise et on juge la vie familiale et sexuelle de la façon la moins compatible avec la moralité catholique, l'expliquent par une transformation majeure du système normatif du Québec, fondé sur la moralité catholique jusque dans les années 1960 :

These differences can be explained, at least in part, by the fact that almost all of Quebec's French-speaking population during the 1960s belonged to the Roman Catholic Church, at a time when local Catholic Church was changing in one important way while failing to change in another equally important one. More specifically, [...] the Church authorities' refusal either to change their doctrine on marriage and sexuality or to allow the laity to play a decisional role in the definition of doctrine and the orientation of pastoral activities gave Roman Catholics a strong motive for abandoning the Christian tradition as the provider of moral guidelines on sexual and family matters; at the 


\section{Marie MOREAU}

same time, the withdrawal of local Roman Catholic authorities from the set of institutions that structured people's lives "from the cradle to the grave" made it possible for Quebecers to actually abandon this tradition (LAPLANTE et al. $2006: 219-220$ ).

En refusant d'évoluer en même temps que les mœurs et les réalités familiales de ses paroissiens, l'Église catholique a fini par les détourner complètement de la morale qu'elle imposait comme ligne de conduite, à la différence des Protestants, qui n'ont pas eu à remettre en question les critères de moralité selon lesquels ils organisaient et envisagaient leurs comportements familiaux. Toutefois, même s'il y contribue grandement, la particularisation du Canada par rapport aux États-Unis n'est pas uniquement le fait du Québec ; les valeurs libérales que laissent apparaître son jugement sur l'évolution des formes familiales, comparativement aux valeurs nettement plus conservatrices du jugement moral des États-Unis, permettent au Canada, même sans la province francophone, de se distinguer de son voisin. La différence du traitement moral des questions familiales et sociales montre que même si la mondialisation permet d'exporter des modèles culturels, sociaux et familiaux, on est encore loin d'une harmonisation idéologique de l'Amérique du nord.

\section{Bibliographie}

ANGUS REID (2007), «Canadians open-minded on relationships and sexual behaviour, but not drugs », http://www.angus-reid.com/wpcontent/uploads/archived-pdf/2007.12.20 Morality.pdf (consulté le 10 juin 2011).

--- , «Americans More Morally Conservative Than Canadians and Britons », http://angusreidglobal.com/wpcontent/uploads/2013/01/2013.01.31_Morality.pdf (consulté le 18 octobre 2015).

BALTHAZAR, Louis (2007), «Vers une incompréhension mutuelle nordaméricaine. Le Canada est-il devenu plus européen qu'américain ? », in C-P. David et J. Tourreille (éds.), Le conservatisme américain : un mouvement qui a transformé les États-Unis, Québec, Presses de l'Université du Québec, pp. 130137.

BEAN, Lydia, MARCO, Gonzalez et KAUFMAN, Jason (2008), "Why Doesn't Canada Have an American-style Christian Right? A Comparative 


\section{LA MORALE À L’ÉPREUVE DE LA MONDIALISATION}

Framework for Analyzing the Political Effects of Evangelical Subculture Identity », Canadian Journal of Sociology 33.4, pp. 899-943.

CDC (Center for Disease Control and Prevention), gouvernement des ÉtatsUnis, « National Marriage and Divorce Rate Trends: 2000-2014 », National Vital Statistics System,

http://www.cdc.gov/nchs/nvss/marriage divorce tables.htm (consulté le 22 février 2015).

HIMMELFARB, Gertrude (2001), One Nation, Two Cultures, 2e éd., New York, Vintage Books.

HOOVER, Dennis R., MARTINEZ, Michael D., REIMER, Samuel H. et WALD, Kenneth D. (2002), «Evangelicalism Meets the Continental Divide: Moral and Economic Conservatism in the United States and Canada », Political Research Quarterly 55.2, pp. 351-374.

LAPLANTE, Benoît, MILLER, Caia et MALHERBE, Paskall (2006), « The Evolution of Beliefs and Opinions on Matters Related to Marriage and Sexual Behaviour among French-Speaking Catholic Quebecers and English-Speaking Protestant Ontarians », Canadian Studies in Population 33.2 vol.33, n², p.209239.

LE BOURDAIS, Céline et MARCIL-GRATTON, Nicole (1996), «Family Transformations across the Canadian/American Border: When the Laggard becomes the Leader », Journal of Comparative Family Studies 27.3, pp. 415436.

LIPSET, Seymour Martin (1996), American Exceptionalism: A Double-Edged Sword, New York, W.W. Norton.

--- (1991), Continental Divide: The Values and Institutions of the United States and Canada, 2e éd., New York, Routledge.

--- (1986), « Historical and National Characteristics: A Comparative Analysis of Canada and the United States », The Canadian Journal of Sociology 11.2, pp. 113-155.

ROSSON, Thomas et FIELDS, Dail (2008), "Cultural Influences on the Growth in Evangelical Christianity: A Longitudinal Study of 49 Countries », Review of Religious Research 49.3, pp. 269-289.

SANDS, Kathleen M. (2000), "Introduction », in K. M. Sands (éd.), God Forbid: Religion and Sex in American Public Life, New York, Oxford University Press, pp. 3-20. 


\section{Marie MOREAU}

SHERIF TRASK, Bahira (2008), Globalization and Families: Accelerated Systemic Social Change, New York, Springer. 